\title{
Superficial Fibromatosis
}

Superficial fibromatosis is a family of fibromatoses of superficial tissues and includes palmar/plantar fibromatosis, penile fibromatosis (also known as Peyronie disease and induratio penis plastica), knuckle pads (also known as Garrod's pads), pachydermodactyly, and infantile digital fibromatosis (also known as Reye's tumor, multiple hyaline fibromatosis).

For more details on palmar/plantar fibromatosis, $\rightarrow$ see dedicated section.

For fibromatosis of the deep tissues (deep fibromatosis), $\rightarrow$ see Chap. 69. 